\title{
3. Introduction to Programming
}

As has already been mentioned, the computer needs to be given a list of instructions in advance telling it how to do any job you want it to do.

So - for example - if you want it to work out the pay figures for your employees then you will have to give it a set of instructions telling it how it can do this. Likewise if you want to do word-processing on a computer - then it will have to be given a suitable set of instructions telling it how it can pretend to be a typewriter for you !

Such a set of instructions is called a program.

These sets of instructions can frequently be bought ready made - in the same way that you can buy a set of instructions (a recipe) telling you how to make a particular type of cake. If so then they will normally come stored on a tape or disk so that you can load them straight onto the machine and let it use them.

Often there is not a set of instructions which exactly matches what is needed. For example, a very large company which wants its computer to keep records of all its stock will probably have different requirements from those of another company so it will need a set of instructions written specially - and if so it will need to employ people (called programmers) to write these instructions.

The instructions can be written in any one or more of a number of languages designed for the purpose - e.g.

COBOL, BASIC, FORTRAN, ALGOL, C, Pascal, etc.

All these languages are similar to English or to mathematical expressions and are therefore fairly easy to learn. However they all have very small vocabularies and the main problem involved in programming is expressing a complicated task as a set of simple steps.

Most languages have specialist uses. For example FORTRAN and ALGOL are used for mathematics and scientific applications, BASIC was originally intended as a beginners' language for training (although it has since become popular for a wide range of jobs), and $\mathrm{COBOL}$ is intended for use in business.

The computer will not be able to follow any of these languages directly but will first have to convert anything written in them into its own language - a set of difficult-to-follow numbers - called machine code (each different type of computer can have its own machine code). This translation is carried out by a program called a compiler - which you would normally be able to buy to convert your chosen language to your own computer's particular machine code. 